\title{
Cardiac Surgery in Germany during 2011: A Report on Behalf of the German Society for Thoracic and Cardiovascular Surgery
}

\author{
Anne-Kathrin Funkat ${ }^{1}$ Andreas Beckmann ${ }^{2}$ Jana Lewandowski ${ }^{2}$ Michael Frie ${ }^{3}$ Wolfgang Schiller ${ }^{4}$ \\ Markus Ernst ${ }^{5}$ Khosro Hekmat ${ }^{6}$
}

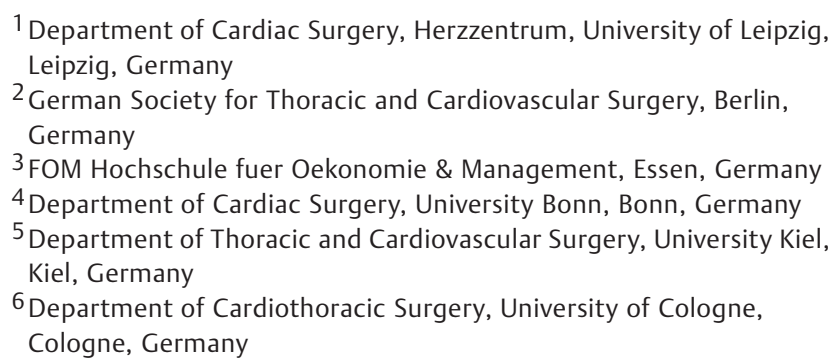

Address for correspondence and reprint requests Anne-Kathrin Funkat, Ph.D., Department of Cardiac Surgery, Herzzentrum, University of Leipzig, Strümpellstr. 39, Leipzig 04289, Germany (e-mail: funkat.ch@herzzentrum-leipzig.de).

Thorac Cardiovasc Surg 2012;60:371-382.

\author{
Abstract \\ Keywords \\ - cardiac surgery \\ - registry \\ - heart valve diseases \\ - coronary heart \\ disease \\ - congenital heart \\ disease \\ - vascular surgery \\ - organ transplantation \\ - heart rhythm \\ disorders
}

\begin{abstract}
All cardiac surgical procedures performed in 78 German cardiac surgical units throughout the year 2011 are presented in this report, based on a voluntary registry which is organized by the German Society for Thoracic and Cardiovascular Surgery. In 2011, a total of 100,291 cardiac surgical procedures (implantable cardioverter defibrillator and pacemakers procedures excluded) have been collected in this registry. More than $13.4 \%$ of the patients were older than 80 years compared with $12.4 \%$ in 2010 . Hospital mortality in 41,976 isolated coronary artery bypass graft procedures ( $14.7 \%$ off-pump) was $2.9 \%$. In 26,972 isolated valve procedures (including 5,210 catheter-based procedures), an in-hospital mortality of $5.2 \%$ has been observed.

This voluntary registry of the German Society for Thoracic and Cardiovascular Surgery will continue to be an important tool enabling quality control and illustrating current facts and the development of cardiac surgery in Germany.
\end{abstract}

\section{Introduction}

Increasing demands for quality control in medicine-by patients, relatives, insurance companies, and authorities all over the world-have stimulated the development of a wide range of registries and other tools to answer those needs. As early as in 1978, the German Society for Thoracic and Cardiovascular Surgery (www.dgthg.de) decided to set up a voluntary registry for cardiac surgical procedures. The aim of this registry continues to illustrate the development of cardiac surgery in Germany and to allow each individual cardiac surgical unit to compare its own results to the nationwide results.

Innovative technologies as minimal invasive mitral valve surgery, off-pump surgery, and still experimental procedures such as transcatheter (transapical or transvascular) aortic valve implantation (TAVI) (- Table V1) have been included in the registry to monitor the development in this field, important for the future of patient care. received

July 27, 2012

accepted

July 27, 2012
Copyright @ 2012 by Thieme Medical Publishers, Inc., 333 Seventh Avenue, New York, NY 10001, USA. Tel: +1(212) 584-4662.
DOI http://dx.doi.org/ 10.1055/s-0032-1326724. ISSN 0171-6425. 
The registry data are published once a year since $1989 .^{1-21}$ The following report contains the assorted data for the year 2011.

\section{Materials and Methods}

Since 2004, the standardized questionnaire asks for detailed information about each individual procedure exactly defined by one or more operation codes (German Operationen- und Prozedurenschlüssel [OPS]).

All centers had to complete the questionnaire until January 20, 2012, asking for all performed procedures and associated hospital mortality in each institution. The completed questionnaires were sent to the office of the German Society for Thoracic and Cardiovascular Surgery in Berlin, and were evaluated for completeness and compiled for further analysis, thus ensuring anonymity for the individual center. The compilation algorithm guaranties a high compliance for submission of the complete dataset.

Inclusion criteria for the registry 2011 were all individual surgical procedures performed between January 1, 2011 and December 31, 2011, unrelated to admission or discharge dates as compared with other registries.

Alike to all previous reports, the number of procedures was counted, not individual patients, for example, a patient requiring additional coronary surgery due to a complication after aortic valve replacement during the same admission would be counted in the category "aortic valve replacement" and in the category "coronary surgery." Thus the registry contains more procedures than the actual number of patients operated on.

Mortality was defined as in-hospital mortality. As per the definition, the observed mortality was attributed to the first heart surgical procedure, for example, the death of a patient requiring additional coronary artery bypass grafting (CABG) due to a complication after aortic valve replacement during the same admission would be attributed only to the aortic valve but not to the coronary surgery group.

The main reason for this structural setup of the registry, like in previous years, was getting detailed information on all performed procedures and not only counting the number of the treated patients. Another reason was to simplify the process of data acquisition thus enabling all cardiac surgery units in Germany, the submission of a complete dataset, regardless of their existing hardware and software.

In 2011, a total of 79 units performed heart surgery, 78 units answered the questionnaire and delivered a complete dataset for the year 2011 including hospital mortality rates. Due to technical reasons, one unit with $\sim 200$ cases could not deliver the data.

\section{Registry Data 2011}

- Table 1 demonstrates the development of procedures using cardiopulmonary bypass (CPB) over the past 30 years in

Table 1 Frequency in Open Heart Procedures in Germany from 1978 to 2011 (1978 to 1985: Federal Republic of Germany [West Germany] Only)

\begin{tabular}{|c|c|c|c|c|c|c|c|c|c|c|c|}
\hline Year & 1978 & 1980 & 1985 & 1990 & 1995 & 2005 & 2007 & 2008 & 2009 & 2010 & 2011 \\
\hline $\begin{array}{l}\text { Total Number } \\
\text { of Units }\end{array}$ & 21 & 21 & 33 & 46 & 76 & 79 & 80 & 79 & 80 & 79 & 78 \\
\hline $\begin{array}{l}\text { Total Number } \\
\text { of Operations }\end{array}$ & 8365 & 10680 & 21705 & 38783 & 78184 & 91967 & 91618 & 89773 & 86916 & 84686 & 84402 \\
\hline Average Per Unit & 398 & 509 & 658 & 843 & 1029 & 1164 & 1145 & 1136 & 1086 & 1072 & 1082 \\
\hline
\end{tabular}

Table 2 Total Results of All 78 Units Performing Cardiac Surgery in 2011

\begin{tabular}{|l|l|l|l|l|}
\hline Category & With CPB & Without CPB & Total & \% Change \\
\hline Valve Procedures & 21887 & 5085 & 26972 & +7.3 \\
\hline Coronary Surgery & 48743 & 6556 & 55299 & -1.2 \\
\hline Congenital Heart Surgery & 4609 & 990 & 5599 & 7201 \\
\hline Surgery of Thoracic Aorta & 6658 & 543 & 2630 & +2.1 \\
\hline Other Cardiac Surgery & 1300 & 1330 & 2109 & +6.4 \\
\hline Assist Devices & 724 & 1385 & 26005 & +1.9 \\
\hline Pacemaker and ICD & 76 & 25929 & 47532 & 173347 \\
\hline Extracardiac Surgery & 405 & 88945 & +4.6 & +8.9 \\
\hline Total & 84402 & +4.0 \\
\hline
\end{tabular}

ICD, implantable cardioverter defibrillator; CPB, cardiopulmonary bypass.

Note: The \% indicates changes compared with 2010. 
Table 3 Distribution of Individual Units According to the Number of Cardiac Surgery Procedures with or without CPB

\begin{tabular}{|l|l|l|l|l|l|}
\hline Number of Operations & $<500$ & $500-999^{a}$ & $1000-1499$ & $1500-1999$ & $2000-5000$ \\
\hline Number of Units & 5 & 25 & 26 & 13 & 9 \\
\hline Average Per Unit & 396 & 777 & 1194 & 1742 & 2802 \\
\hline Minimum-Maximum & $245-477$ & $510-974$ & $1002-1458$ & $1516-1968$ & $2075-3823$ \\
\hline
\end{tabular}

CPB, cardiopulmonary bypass.

${ }^{\mathrm{a}}$ One unit is performing pediatric cardiac surgery only.

Table 4 Distribution of Units According to Surgical Profiles in 2011

\begin{tabular}{|l|l|}
\hline Type of Surgery Performed & Number of Units \\
\hline Coronary Surgery & 77 \\
\hline Valve Surgery & 77 \\
\hline Surgery of Congenital Heart Diseases With CPB in Children < 1 Year & $26^{\mathrm{a}}$ \\
\hline Heart Transplantation & $22^{\mathrm{b}}$ \\
\hline Heart-Lung Transplantation & 5 \\
\hline
\end{tabular}

$\mathrm{CPB}$, cardiopulmonary bypass.

a Surgery for congenital heart disease with CPB in children < 1 year (n 2037); thereof: 2 to 18 operations in five units, 26-45 operations in five units, 52-84 operations in eight units, and 109-250 operations in eight units.

bHeart transplantations ( $n=355)$ : $70 \%$ of the total annual heart transplantations are performed by 8 of 22 units with $\geq 15$ heart transplantation per year; thereof: 1-4 transplants in three units, 5-9 transplants in six units, 10-19 transplants in seven units, and 21-77 transplants in six units.

Table 5 Additional Demographic Data for Procedures with CPB in 2011 and 2010

\begin{tabular}{|l|l|l|l|l|}
\hline Demographic Data & \multicolumn{2}{|c|}{2011} & \multicolumn{2}{c|}{2010} \\
\hline Emergency Operations & 11911 & $11.9 \%$ & 11850 & $12.0 \%$ \\
\hline Redo Procedures & 8511 & $8.5 \%$ & 8458 & $8.6 \%$ \\
\hline Age $>69$ Years $^{\mathrm{a}}$ & 95456 & $53.6 \%$ & 93646 & $52.2 \%$ \\
\hline
\end{tabular}

$\mathrm{CPB}$, cardiopulmonary bypass.

Note: The numbers in each category reflect procedures and not individual patients.

${ }^{a}$ Without patients younger than 20 years.

Germany. The number of heart operations procedures with CPB remains on a stable level.

Overall, 173,347 procedures were reported to the registry for the year 2011, an increase of 4.0\% (2010: 166,621 procedures). A total of 100,291 cardiac surgical procedures (excluded: implantable cardioverter defibrillator (ICD), pacemakers, and miscellaneous procedures without $\mathrm{CPB}$ ) display an increase of $1.74 \%$ ( $n=1,714)$ compared with the year of 2010 (98,755 procedures) (-Table 2 ). The following tables and figures (-Tables $\mathbf{3}$ to $\mathbf{6}$, - Tables V1 to V7, - Tables C1 to C3, - Tables Con1 and Con2, - Tables Mis1 to Mis5 and - Figs. 1 to $\mathbf{1 1}$ ) represent the compiled registry data for 2011 classified in individual categories.

As in previous years, several important developments continued also in 2011. Over the past 18 years, the age distribution (-Fig. 6) demonstrates a shift to the categories with older patients. Currently, $53.6 \%$ of the cardiac procedures are performed in patients 70 years or older and $13.4 \%$ in patients 80 years or older. However, mortality remains the
Table 6 Gender Distribution

\begin{tabular}{|l|l|l|}
\hline \multicolumn{3}{|l|}{ Male/Female Ratio among Cardiac Procedures } \\
\hline Heart Valve Procedures & $55 \%$ & $45 \%$ \\
\hline Coronary Surgery & $75 \%$ & $25 \%$ \\
\hline Congenital Heart Surgery & $52 \%$ & $48 \%$ \\
\hline Surgery of Thoracic Aorta & $68 \%$ & $32 \%$ \\
\hline Other Cardiac Surgery & $44 \%$ & $56 \%$ \\
\hline Assist Devices & $71 \%$ & $29 \%$ \\
\hline Pacemaker and ICD & $59 \%$ & $41 \%$ \\
\hline Extracardiac Surgery & $63 \%$ & $37 \%$ \\
\hline Total & $65 \%$ & $35 \%$ \\
\hline
\end{tabular}

ICD, implantable cardioverter defibrillator.

Note: All coronary surgery (48743 on-pump and 6556 off-pump procedures) and all congenital surgery procedures are included in this table. 
374 Cardiac Surgery in Germany during 2011 Funkat et al.

Table V1 Single Heart Valve Procedures

\begin{tabular}{|l|l|l|l|}
\hline Position & $\boldsymbol{n}$ & Deaths & \% \\
\hline Aortic Sternotomy & 9874 & 331 & 3.4 \\
\hline Aortic Part. Sternotomy & 1794 & 49 & 2.7 \\
\hline Aortic Endovascular & 2306 & 117 & 5.1 \\
\hline Aortic Transapical & 2777 & 241 & 8.7 \\
\hline Mitral Sternotomy & 3231 & 193 & 6.0 \\
\hline Mitral Mic & 2280 & 56 & 2.5 \\
\hline Mitral Transcatheter & 120 & 5 & 4.2 \\
\hline Tricuspidal Sternotomy & 363 & 38 & 10.5 \\
\hline Tricuspidal Mic & 92 & 5 & 5.4 \\
\hline Pulmonary Sternotomy & 42 & 1 & 2.4 \\
\hline Pulmonary Mic & 0 & - & - \\
\hline Pulmonary Transcatheter & 1 & 0 & 0.0 \\
\hline Total & 22880 & 1036 & 4.5 \\
\hline
\end{tabular}

part., partial; mic, minimally invasive surgery.

Note: A total of $2280(41 \%)$ mitral valve procedures were done using a minimally invasive access. The number of isolated conventional aortic valve replacement showed no difference from 2010 to 2011 (2010: 11689 and 2011:11668 procedures).

Table V2 Isolated Aortic Valve Procedures

\begin{tabular}{|l|l|l|l|}
\hline Type of Valve & $\boldsymbol{n}$ & Deaths & \% \\
\hline Prosthesis & 1689 & 41 & 2.4 \\
\hline Xenograft & 9846 & 334 & 3.4 \\
\hline Homograft & 37 & 2 & 5.4 \\
\hline Reconstruction & 96 & 3 & 3.1 \\
\hline Total & 11668 & 380 & 3.3 \\
\hline
\end{tabular}

Abbreviation: transcatheter aortic valve implantation.

Note: Out of 11668 procedures, 1794 (15\%) were done by a partial sternotomy access. TAVI excluded.

Table V3 Isolated Heart Valve Procedures

\begin{tabular}{|l|l|l|l|}
\hline Procedures & $\boldsymbol{n}$ & Deaths & \% \\
\hline Single & 17676 & 673 & 3.8 \\
\hline Double & 3600 & 299 & 8.3 \\
\hline Triple & 362 & 53 & 14.6 \\
\hline Transcatheter Access & 5210 & 363 & 7.0 \\
\hline Not Specified & 124 & 10 & 8.1 \\
\hline Total & 26972 & 1398 & 5.2 \\
\hline
\end{tabular}

Note: Combined procedures (coronary artery bypass graft and aortic surgery) are not included.

same or even decreased slightly over the past 18 years (see - Fig. 2). Although the number of CABG decreased while the number of off-pump procedures for coronary heart disease increased to $14.7 \%$ (2010: $14.2 \%$ ) (-Fig. 3 ).
Table V4 Isolated Mitral Valve Procedures

\begin{tabular}{|l|l|l|l|}
\hline Type of Valve & $\boldsymbol{n}$ & Deaths & $\%$ \\
\hline Prosthesis & 579 & 28 & 4.8 \\
\hline Xenograft & 1334 & 152 & 11.4 \\
\hline Homograft & 11 & 3 & 27.3 \\
\hline Reconstruction & 3587 & 66 & 1.8 \\
\hline Total & 5511 & 249 & 4.5 \\
\hline
\end{tabular}

Note: Out of 5511 procedures, 2280 (41\%) were done using a minimally invasive access. Transcatheter procedures excluded.

Table V5 Multiple heart Valve Procedures

\begin{tabular}{|l|l|l|l|}
\hline Combination & $\boldsymbol{n}$ & Deaths & $\%$ \\
\hline Aortic + Mitral & 1854 & 153 & 8.3 \\
\hline Mitral + Tricuspid & 1419 & 110 & 7.8 \\
\hline Aortic + Tricuspid & 188 & 33 & 17.6 \\
\hline Tricuspid + Pulmonary & 17 & 2 & 11.8 \\
\hline Aortic + Pulmonary & 122 & 1 & 0.8 \\
\hline Aortic + Mitral + Tricuspid & 357 & 50 & 14.0 \\
\hline Aortic + Mitral + Pulmonary & 5 & 3 & 60.0 \\
\hline Total & 3962 & 352 & 8.9 \\
\hline
\end{tabular}

Note: Transcatheter procedures are not included.

ancluding Ross procedures.

Since 2004 more than $50 \%$ of isolated mitral valve procedures are reconstructions, in 2011 more than $65 \%$ of the patients with mitral valve disease received a mitral valve reconstruction (-Fig. 8). For correct interpretation of $\boldsymbol{\sim}$ Fig. $\mathbf{8}$, it is important to assert that, due to the data collection method (German OPS), all patients with various mitral valve diseases such as mitral valve stenosis, valve calcification, endocarditis, and patients under emergent conditions are included. The operation codes by itself give no information about the underlying disease. The reconstruction rate would certainly be higher if only patients would be included where a reconstruction is feasible. In other publications, for example, Gammie et al, ${ }^{22}$ the reconstruction rate must be interpreted very carefully compared with our registry data because in their publication, patients with mitral valve stenosis, endocarditis, and in emergent conditions are excluded.

The increase of left ventricular assist device implantation ( -Fig. 10) emphasizes the increasing relevance of mechanical circulatory support.

The most remarkable evolution is the extensive increase of TAVI over the past 6 years ( $\mathbf{F i g}$. $\mathbf{5}$ ), while the number of isolated aortic valve replacements by open surgery remains stable. Starting in 2006 with 78 procedures ( $0.67 \%$ of isolated aortic valve procedures), 5,083 TAVI are reported in 2011 (30.5\%). However, it should be kept in mind that the 78 units which contribute at this registry are not the only sites which are performing TAVI in Germany. TAVI via transvascular access are also performed in cardiology units without 
Cardiac Surgery in Germany during 2011 Funkat et al. 375

Table V6 Mitral Valve Surgery-Implantation/Replacement Versus Reconstruction

\begin{tabular}{|l|l|l|l|l|l|l|l|l|l|l|}
\hline \multirow{2}{*}{ Mitral Valve Surgery } & & & & & \multicolumn{3}{|c|}{ Replacement } & \multicolumn{3}{c|}{ Reconstruction } \\
\cline { 2 - 11 } & $n$ & $\begin{array}{l}\text { Total } \\
\text { Deaths }\end{array}$ & $\begin{array}{l}\% \\
\text { Death }\end{array}$ & $\begin{array}{l}\text { Reconstruction } \\
\text { Reath }\end{array}$ & $\boldsymbol{n}$ & Deaths & \% Death & Deaths & Death \\
\hline Isolated & 5511 & 249 & 4.5 & 65.1 & 1924 & 183 & 9.5 & 3587 & 66 & 1.8 \\
\hline Mitral Valve + & & & & & & & & & & \\
\hline Aortic Valve & 1854 & 153 & 8.3 & 62.8 & 689 & 103 & 14.9 & 1165 & 50 & 4.3 \\
\hline $\begin{array}{l}\text { Tricuspid Valve } \\
\text { Reconstruction }\end{array}$ & 1390 & 104 & 7.5 & 64.3 & 496 & 55 & 11.1 & 894 & 49 & 5.5 \\
\hline CABG & 2590 & 269 & 10.4 & 68.6 & 812 & 139 & 17.1 & 1778 & 130 & 7.3 \\
\hline $\begin{array}{l}\text { CABG + Aortic Valve } \\
\text { Replacement }\end{array}$ & 957 & 114 & 11.9 & 70.7 & 280 & 52 & 18.6 & 677 & 62 & 9.2 \\
\hline Total & 12302 & 889 & 7.2 & 65.9 & 4201 & 532 & 12.7 & 8101 & 357 & 4.4 \\
\hline
\end{tabular}

CABG, coronary artery bypass graft.

${ }^{a}$ Twenty-nine procedures (not specified Mitral valve + tricuspid valve surgery) excluded. Deaths \%: 20.7 (6/29).

Table V7 Transcatheter Heart Valve Procedures: 54.6\% of TAVI were Done Using a Transapical Access

\begin{tabular}{|c|c|c|c|c|c|c|c|}
\hline & \multirow[b]{2}{*}{ Total } & \multirow[b]{2}{*}{ Deaths } & \multirow[b]{2}{*}{ Death \% } & \multicolumn{2}{|c|}{ With CPB } & \multicolumn{2}{|c|}{ Without CPB } \\
\hline & & & & $n$ & Deaths & $n$ & Deaths \\
\hline Aortic Valve Implantation & 5083 & 358 & 7.0 & 121 & 42 & 4962 & 316 \\
\hline Transvascular Access ${ }^{\mathrm{a}}$ & 2306 & 117 & 5.1 & 34 & 13 & 2272 & 104 \\
\hline Transapical Access & 2777 & 241 & 8.7 & 87 & 29 & 2690 & 212 \\
\hline Mitral Valve & 120 & 5 & 4.2 & 2 & 0 & 118 & 5 \\
\hline Repair & 94 & 1 & 1.1 & 1 & 0 & 93 & 1 \\
\hline Implantation $^{\mathrm{b}}$ & 26 & 4 & 15.4 & 1 & 0 & 25 & 4 \\
\hline Aortic Valve Implantation ${ }^{\mathrm{b}}+\mathrm{CABG}$ & 38 & 8 & 21.1 & 15 & 5 & 23 & 3 \\
\hline Total & 10444 & 734 & 7.0 & 261 & 89 & 10183 & 645 \\
\hline
\end{tabular}

TAVI, transcatheter aortic valve implantation; CPB, cardiopulmonary bypass; CABG, coronary artery bypass graft.

Note: Pulmonary valve implantation for the correction of congenital lesions are not included, one procedure was reported for adults without congenital lesion. With the use of CPB, 2.3\% of TAVI procedures were performed. It has to be assumed that CPB was mostly used in emergency situations, which explains the lethality of $34.7 \%$ in this group. Nevertheless, this underlines the necessity of a fully equipped surrounding for TAVI procedures where CPB is on standby for urgent use.

${ }^{a}$ Femoral, subclavian or transaortic access.

${ }^{\mathrm{b}}$ Endovascular and transapical access.

Table C1 Isolated CABG with CPB and Combined Procedures with CPB

\begin{tabular}{|l|l|l|l|}
\hline Procedures & $n$ & Deaths & \% \\
\hline CABG & 41976 & 1217 & 2.9 \\
\hline CABG+ & & 0 & \\
\hline TMLR & 4 & 18 & 0.0 \\
\hline Aneurysm Resection & 230 & 441 & 7.8 \\
\hline Aortic Valve Replacement & 8023 & 8 & 5.5 \\
\hline Transcatheter Aortic Valve Implantation & 38 & 139 & 21.1 \\
\hline Mitral Valve Replacement & 812 & 130 & 17.1 \\
\hline Mitral Valve Repair & 1778 & 52 & 7.3 \\
\hline Aortic + Mitral Valve Replacement & 280 & 62 & 18.6 \\
\hline Aortic Valve Replacement + Mitral Valve Repair & 677 & 102 & 9.2 \\
\hline Other & 1481 & 2169 & 6.9 \\
\hline Total & 55299 & & \\
\hline
\end{tabular}

CABG, coronary artery bypass graft; $C P B$, cardiopulmonary bypass. 
376 Cardiac Surgery in Germany during 2011 Funkat et al.

Table C2 Isolated CABG with CPB

\begin{tabular}{|l|l|l|l|}
\hline Number of Grafts & $\boldsymbol{n}$ & Deaths & \% \\
\hline Single & 1126 & 66 & 5.9 \\
\hline Double & 7748 & 226 & 2.9 \\
\hline Triple & 15749 & 489 & 3.1 \\
\hline Quadruple & 8767 & 236 & 2.7 \\
\hline Quintuple + more & 2431 & 71 & 2.9 \\
\hline Total & 35821 & 1088 & 3.0 \\
\hline
\end{tabular}

CABG, coronary artery bypass graft; CPB, cardiopulmonary bypass.

Table C3 Off-Pump Isolated CABG

\begin{tabular}{|l|l|l|l|}
\hline Number of Grafts & $\boldsymbol{n}$ & Deaths & $\%$ \\
\hline Single & 1514 & 34 & 2.2 \\
\hline Double & 1807 & 53 & 2.9 \\
\hline Triple & 2004 & 33 & 1.6 \\
\hline Quadruple & 708 & 8 & 1.1 \\
\hline Quintuple + More & 122 & 1 & 0.8 \\
\hline Total & 6155 & 129 & 2.1 \\
\hline
\end{tabular}

CABG, coronary artery bypass graft.
Table Con1 Age Distribution among Procedures for Congenital Heart Disease

\begin{tabular}{|c|c|c|c|}
\hline Age & $n$ & Deaths & $\%$ \\
\hline \multicolumn{4}{|l|}{ Without CPB } \\
\hline Over 18 Years & 79 & 2 & 2.5 \\
\hline $1-17$ Years & 178 & 1 & 0.6 \\
\hline Under 1 Year & 733 & 11 & 1.5 \\
\hline Total of $A$ & 990 & 14 & 1.4 \\
\hline \multicolumn{4}{|l|}{ With CPB } \\
\hline Over 18 Years & 878 & 25 & 2.8 \\
\hline $1-17$ Years & 1694 & 8 & 0.5 \\
\hline Under 1 Year & 2037 & 73 & 3.6 \\
\hline Total of B & 4609 & 106 & 2.3 \\
\hline
\end{tabular}

CPB, cardiopulmonary bypass.

availability of a heart surgical unit. With the use of CPB, 2.3\% of TAVI procedures were performed. It has to be assumed that CPB was mostly used in emergency situations, which explains the lethality of $34.7 \%$ in this group. Nevertheless, this underlines the necessity of a fully equipped surrounding for TAVI procedures where CPB is on standby for urgent use.

Table Con2 Procedures for Congenital Heart Disease with and without CPB

\begin{tabular}{|c|c|c|c|c|c|c|c|c|c|}
\hline \multirow[t]{2}{*}{ Lesion } & \multicolumn{3}{|c|}{ Age $<1$ Year } & \multicolumn{3}{|c|}{ Age $1-17$ Years } & \multicolumn{3}{|c|}{ Age $\geq 18$ Years } \\
\hline & $n$ & Deaths & $\%$ & $n$ & Deaths & $\%$ & $n$ & Deaths & $\%$ \\
\hline ASD & 74 & 0 & 0.0 & 279 & 0 & 0.0 & 271 & 6 & 2.2 \\
\hline Complete AV Canal & 180 & 5 & 2.8 & 76 & 0 & 0.0 & 14 & 1 & 7.1 \\
\hline VSD & 303 & 1 & 0.3 & 121 & 0 & 0.0 & 26 & 1 & 3.8 \\
\hline Fallot's Tetralogy & 206 & 5 & 2.4 & 36 & 1 & 2.8 & 6 & 0 & 0.0 \\
\hline DORV & 65 & 1 & 1.5 & 14 & 0 & 0.0 & 0 & 0 & - \\
\hline TGA & 132 & 4 & 3.0 & 7 & 0 & 0.0 & 0 & 0 & - \\
\hline $\mathrm{TGA}+\mathrm{VSD}$ & 65 & 2 & 3.1 & 7 & 0 & 0.0 & 0 & 0 & - \\
\hline Truncus Arteriosus & 27 & 2 & 7.4 & 8 & 0 & 0.0 & 0 & 0 & - \\
\hline Fontan & 5 & 0 & 0.0 & 275 & 1 & 0.4 & 10 & 2 & 20.0 \\
\hline Norwood Type & 162 & 21 & 13.0 & 4 & 1 & 25.0 & 0 & 0 & - \\
\hline Pulmonary Valve & 70 & 1 & 1.4 & 238 & 1 & 0.4 & 75 & 2 & 2.7 \\
\hline Transcatheter Pulmonary Valve Implantation & 0 & 0 & - & 7 & 1 & 14.3 & 7 & 0 & 0.0 \\
\hline Aortic Valve & 55 & 4 & 7.3 & 185 & 1 & 0.5 & 305 & 5 & 1.6 \\
\hline Ross Procedure & 12 & 2 & 16.7 & 28 & 0 & 0.0 & 25 & 1 & 4.0 \\
\hline Mitral Valve & 39 & 4 & 10.3 & 77 & 0 & 0.0 & 74 & 7 & 9.5 \\
\hline Tricuspid Valve & 66 & 0 & 0.0 & 47 & 0 & 0.0 & 44 & 0 & 0.0 \\
\hline PDA & 282 & 5 & 1.8 & 20 & 0 & 0.0 & 5 & 0 & 0.0 \\
\hline Coarctation & 184 & 2 & 1.1 & 45 & 0 & 0.0 & 4 & 0 & 0.0 \\
\hline Heart Transplantation & 4 & 0 & 0.0 & 18 & 0 & 0.0 & 0 & 0 & - \\
\hline Heart-Lung Transplantation & 0 & 0 & - & 0 & 0 & - & 0 & 0 & - \\
\hline Lung Transplantation & 0 & 0 & - & 9 & 0 & 0.0 & 0 & 0 & - \\
\hline Others & 839 & 30 & 3.6 & 371 & 11 & 3.0 & 91 & 2 & 2.2 \\
\hline Total & 2770 & 89 & 3.2 & 1872 & 17 & 0.9 & 957 & 27 & 2.8 \\
\hline
\end{tabular}

ASD, atrial septal defect; AV, atrioventricular; VSD, ventricular septal defect; DORV, double outlet right ventricle; TGA, transposition at the great arteries; PDA, patent ductus arteriosus. 
Table Mis1 Development of Ross Procedures in Various Age Groups

\begin{tabular}{|l|l|l|l|l|l|l|l|l|l|l|}
\hline $\begin{array}{l}\text { Autologous Aortic } \\
\text { Valve Replacement } \\
\text { (Ross Procedure) }\end{array}$ & $n$ (2002) & $n$ (2003) & $n$ (2004) & $n$ (2005) & $n$ (2006) & $n$ (2007) & $n$ (2008) & $n$ (2009) & $n$ (2010) & $n$ (2011) \\
\hline In Patients $\geq 18$ Years & 163 & 170 & 250 & 235 & 228 & 261 & 207 & 175 & 184 & 134 \\
\hline In Patients $<18$ Years & 61 & 37 & 50 & 46 & 50 & 34 & 42 & 54 & 43 & 40 \\
\hline Total & 224 & 207 & 300 & 281 & 278 & 295 & 249 & 229 & 227 & 174 \\
\hline
\end{tabular}

Table Mis2 Transplantation All Pediatric Transplantations (Demonstrated in Table Con2) Are Included in This Table

\begin{tabular}{|l|l|l|l|l|l|l|}
\hline \multirow{2}{*}{ Transplantation } & \multicolumn{3}{|c|}{ With CPB } & \multicolumn{3}{c|}{ Without CPB } \\
\cline { 2 - 7 } & $n$ & Deaths & $\%$ & $n$ & Deaths \\
\hline Heart & 355 & 36 & 10.1 & & & \\
\hline Heart + Lung & 10 & 0 & 0.0 & & & \\
\hline Lung & 61 & 8 & 13.1 & 238 & 13 \\
\hline
\end{tabular}

Note: Eurotransplant (ET) has reported for the same period 341 heart transplantations (HTx), 13 heart + kidney transplantations, 2 heart + liver transplantations, 10 heart-lung transplantations, 268 double lung, 57 single lung transplantations (LuTx), 1 lung + kidney transplantations, and 1 lung + liver transplantations. The differences (ET: $-28 \mathrm{LuTx},-1 \mathrm{HTx}$ ) may be explained by different inclusion criteria (time of transplantation) for the registry and the ET database.

$\mathrm{CPB}$, cardiopulmonary bypass.

In the context of TAVI, two other quality assurance initiatives in Germany will be of great interest: the German Aortic Valve Registry and the legal quality assurance ( $\$ 137$ SGB V), which the AQUA-Institute is responsible for.

\section{Discussion}

This report enables a comprehensive overview of all cardiac surgical procedures performed in Germany in 2011. The

Table Mis3 Aortic Surgery

\begin{tabular}{|c|c|c|c|c|c|c|}
\hline \multirow[t]{2}{*}{ Aortic Surgery ${ }^{a}$} & \multicolumn{3}{|c|}{ With CPB } & \multicolumn{3}{|c|}{ Without CPB } \\
\hline & $n$ & Deaths & $\%$ & $n$ & Deaths & $\%$ \\
\hline Supracoronary Ascending & 1523 & 88 & 5.8 & & & \\
\hline Infracoronary Ascending & & & - & & & \\
\hline Mechanical Valve Conduits & 623 & 40 & 6.4 & & & \\
\hline Biological Valve Conduits & 681 & 76 & 11.2 & & & \\
\hline David & 477 & 7 & 1.5 & & & \\
\hline Yacoub & 130 & 4 & 3.1 & & & \\
\hline Other & 277 & 21 & 7.6 & & & \\
\hline Supracoronary Ascending + Aortic Valve Replacement & 1331 & 56 & 4.2 & & & \\
\hline Aortic Arch Replacement ${ }^{b}$ & 1419 & 165 & 11.6 & & & \\
\hline Descending & 105 & 13 & 12.4 & 16 & 4 & 25.0 \\
\hline Thoracoabdominal & 78 & 23 & 29.5 & 31 & 6 & 19.4 \\
\hline Endostent Descending & 14 & 0 & 0.0 & 496 & 27 & 5.4 \\
\hline Total & 6658 & 493 & 7.4 & 543 & 37 & 6.8 \\
\hline
\end{tabular}

Note: All procedures involving aortic surgery are included in this table. Isolated aortic surgery as well as all possible combined procedures (e.g., additional $(A B G)$ are summarized in this category.

${ }^{a}$ Abdominal aortic surgery is not included: 580 abdominal and 467 endostent abdominal.

${ }^{\mathrm{b}}$ All possible combined procedures are included in this category; the only common denominator is aortic arch surgery.

$\mathrm{CPB}$, cardiopulmonary bypass; CABG, coronary artery bypass graft. 
Table Mis4 Pacemaker and ICD Procedures

\begin{tabular}{|c|c|c|c|c|c|c|c|}
\hline \multirow[t]{2}{*}{ Pacemaker and ICD } & \multirow[b]{2}{*}{ Total } & \multirow[b]{2}{*}{ Deaths } & \multirow[b]{2}{*}{ Death \% } & \multicolumn{2}{|c|}{ With CPB } & \multicolumn{2}{|c|}{ Without CPB } \\
\hline & & & & $n$ & Deaths & $n$ & Deaths \\
\hline Pacemaker: Implantation & 9223 & 59 & 0.6 & 3 & 0 & 9220 & 59 \\
\hline Pacemaker: Battery Exchange & 1974 & 2 & 0.1 & 3 & 0 & 1971 & 2 \\
\hline Pacemaker: Revision & 2828 & 12 & 0.4 & 39 & 0 & 2789 & 12 \\
\hline ICD: Implantation & 5380 & 14 & 0.3 & 2 & 0 & 5378 & 14 \\
\hline ICD: Battery Exchange & 1946 & 3 & 0.2 & 0 & 0 & 1946 & 3 \\
\hline ICD: Revision & 2853 & 23 & 0.8 & 22 & 2 & 2831 & 21 \\
\hline Miscellaneous & 651 & 2 & 0.3 & 2 & 0 & 649 & 2 \\
\hline Total & 24855 & 115 & 0.5 & 71 & 2 & 24784 & 113 \\
\hline
\end{tabular}

ICD, implantable cardioverter defibrillator; CPB, cardiopulmonary bypass.

Table Mis5 Surgical Ablation Procedures

\begin{tabular}{|l|l|l|l|}
\hline \multirow{2}{*}{ Energy } & Total & Endocardiac Ablation & Epicardiac Ablation \\
\cline { 2 - 4 } & & $n$ & $n$ \\
\hline Unipolar Radiofrequency & 315 & 283 & 32 \\
\hline Unipolar Cryo-Radiofrequency & 532 & 327 & 205 \\
\hline Bipolar Radiofrequency & 1621 & 193 & 1428 \\
\hline Cryothermy & 1494 & 1136 & 358 \\
\hline Microwave & 56 & 8 & 48 \\
\hline Focused Ultrasound & 465 & 48 & 417 \\
\hline Laser & 0 & 0 & 0 \\
\hline Other & 19 & 1 & 18 \\
\hline Total & 4502 & 1996 & 2506 \\
\hline
\end{tabular}

Note: Included in this table are all isolated ablation procedures and all possible combination procedures (e.g., CABG + ablation). Total of $n=343$ procedures are not specified with regard to endocardiac/epicardiac ablation.

CABG, coronary artery bypass graft.

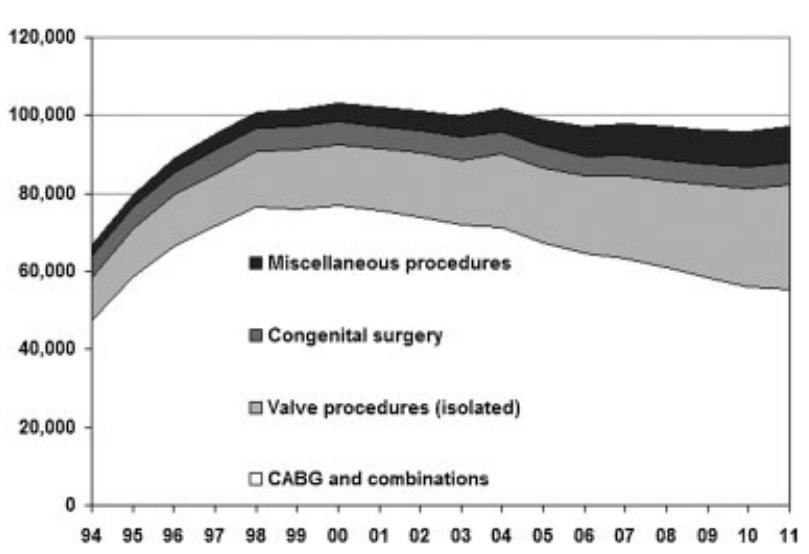

Figure 1 Development of cardiac surgery in Germany from 1994 to 2011. Coronary surgery and combined procedures include all types of isolated coronary surgery with or without CPB and any combined procedure. Heart valve procedures include all types of isolated valve surgery. Combinations of aortic surgery and heart valve procedures are summarized in the miscellaneous group. Congenital heart surgery includes all types of procedures with or without CPB. ASD repair in adults in combination with coronary or heart valve surgery are summarized in the coronary or heart valve surgery group. Miscellaneous includes all other types of procedures with CPB. ASD, atrial septal defect; CPB, cardiopulmonary bypass. accuracy of this registry is reliable due to the implemented compilation algorithm using operation codes. This assumption is supported by other authors who could demonstrate a high accuracy for major outcome parameters in unaudited registries. ${ }^{23}$ Alike to previous reports, we can conclude that cardiac surgery is performed on a high level with a low inhospital mortality compared with other international registries. This conclusion is important especially in the era of continuously increasing patient age combined with relevant comorbidities, both leading to a higher perioperative risk profile.

Compared with 2010, the number of cardiac surgical procedures has stabilized due to the high volume of TAVI.

Further improvements for the structure of the registry are necessary to allow a more detailed and risk adjusted analysis of the collected data. However, significant structural changes of the registry have to ensure data compatibility to allow further longitudinal data analysis.

The future of this voluntary registry as well as its further development will depend on continuous efforts of each individual cardiac surgical unit. This will be of outstanding importance to guarantee the ongoing high quality of cardiac surgery in Germany. 


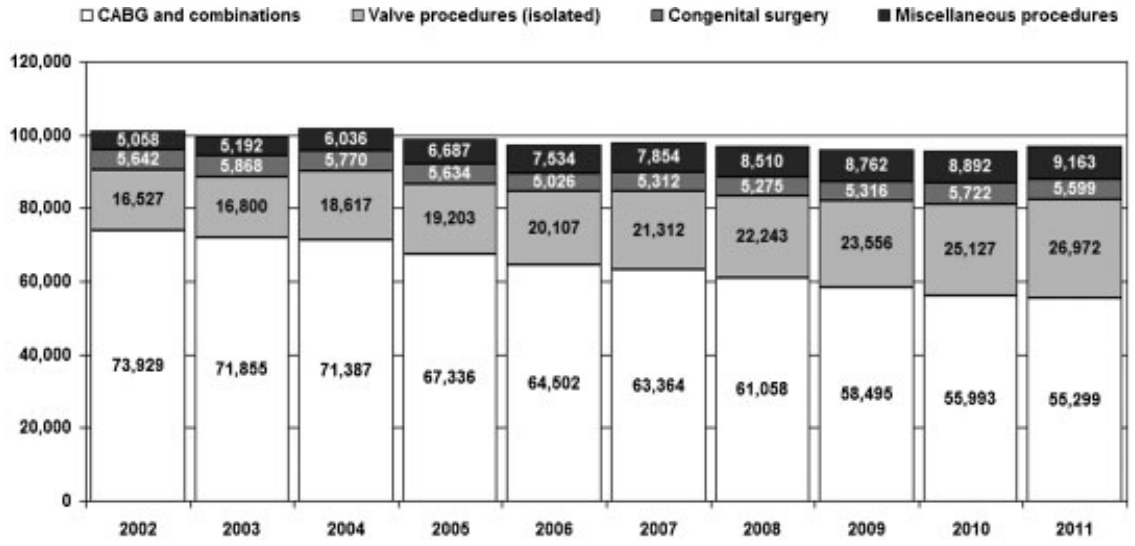

Figure 2 Development of cardiac surgery in Germany during the past 10 years.

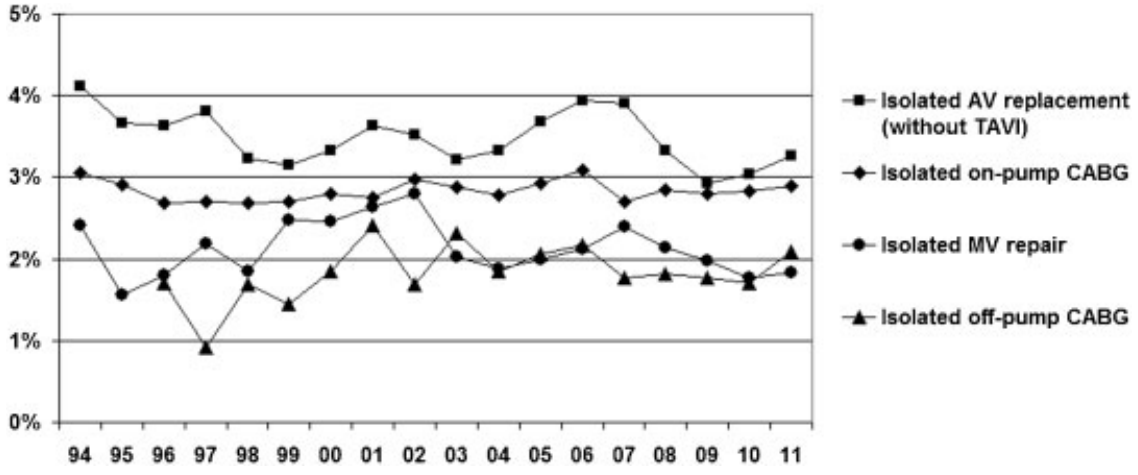

Figure 3 Development of mortality for selected procedures.

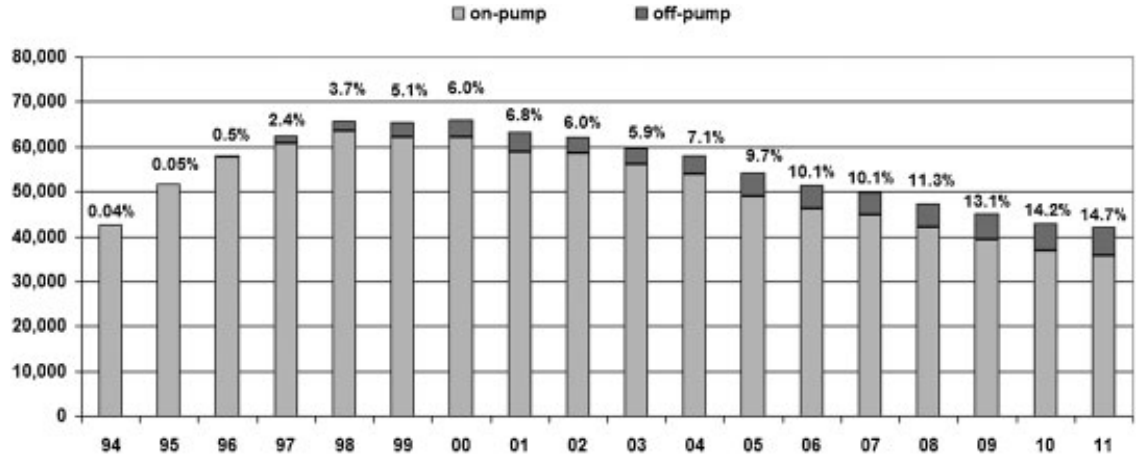

Figure 4 Isolated coronary bypass surgery. The number of coronary artery bypass procedures declined since the year 2000. The percentage of offpump procedures has slightly increased compared with previous years but still has not reached the quantity of other comparable countries.

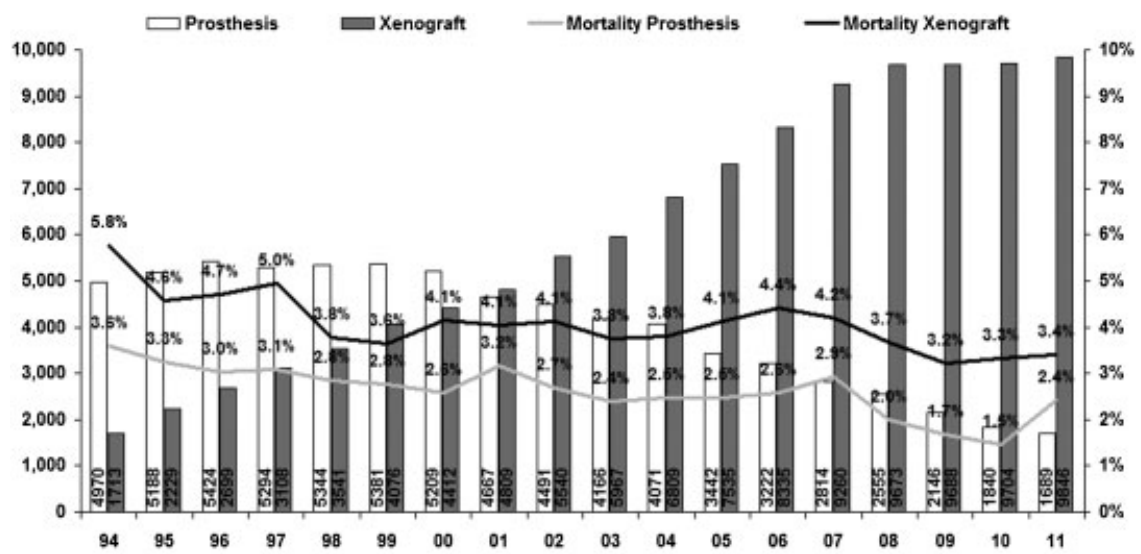

Figure 5 Isolated aortic valve replacement from 1994 to 2011 in Germany. The use of xenografts is steadily increasing. There is a remarkable difference in mortality which is probably age related. Ross or other homograft procedures and transcatheter valve implantations are excluded in this overview. 


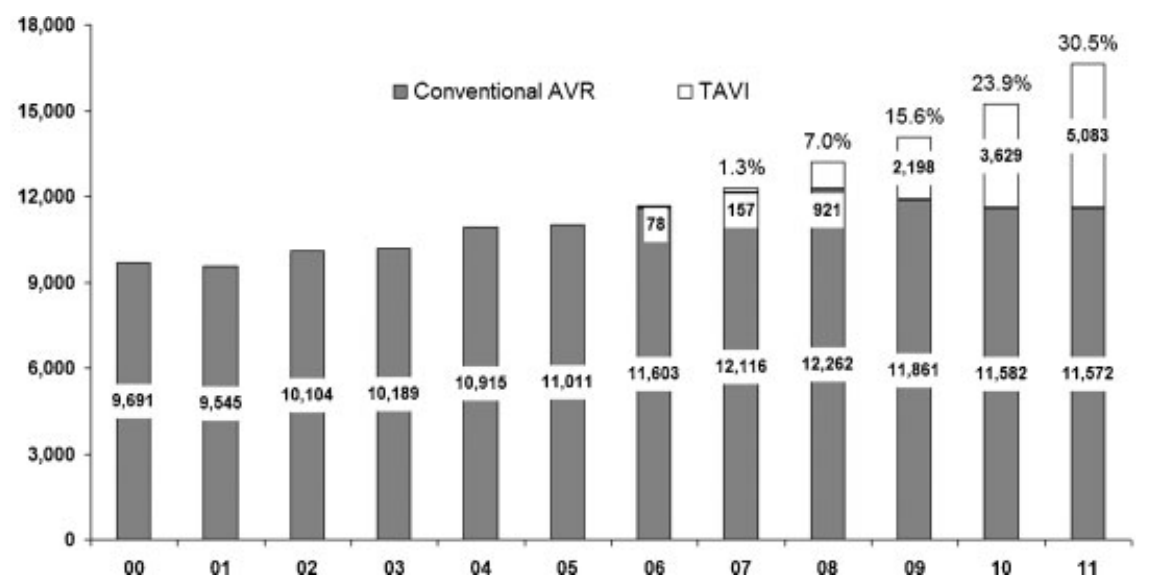

Figure 6 Conventional isolated aortic valve replacement or catheter-based procedures. The figure shows a significant increase in catheter-based procedures. In 2011, more than $30 \%$ of isolated aortic valve procedures were performed using an endovascular or transapical approach. This development underlines the importance of the new founded national aortic valve registry to get valid information about this relatively new therapy.

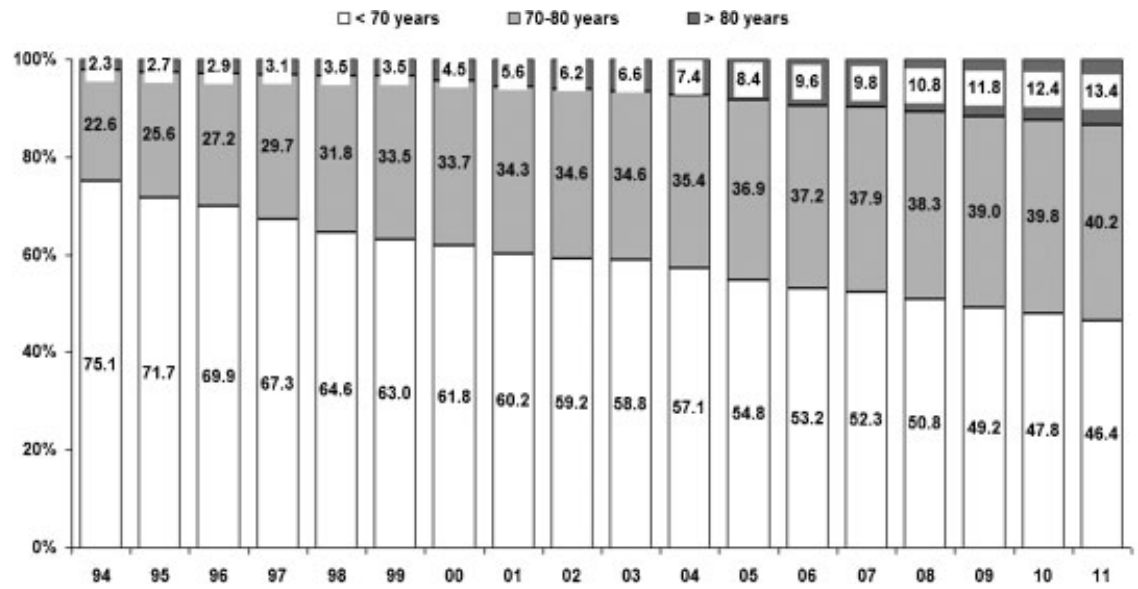

Figure 7 Age distribution of cardiac procedures (without ICD and pacemaker procedures) since 1994. Currently, more than 53\% of the patients are older than 70 years. Patients younger than 20 years are excluded. ICD, implantable cardioverter defibrillator.

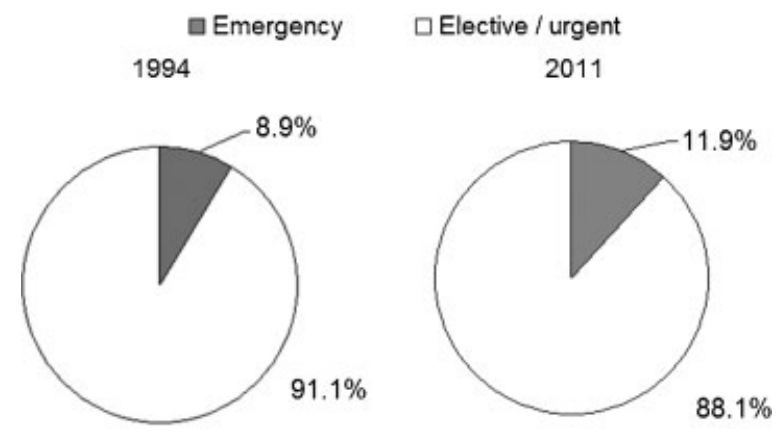

Figure 8 Distribution of urgency 1994 and 2011. 


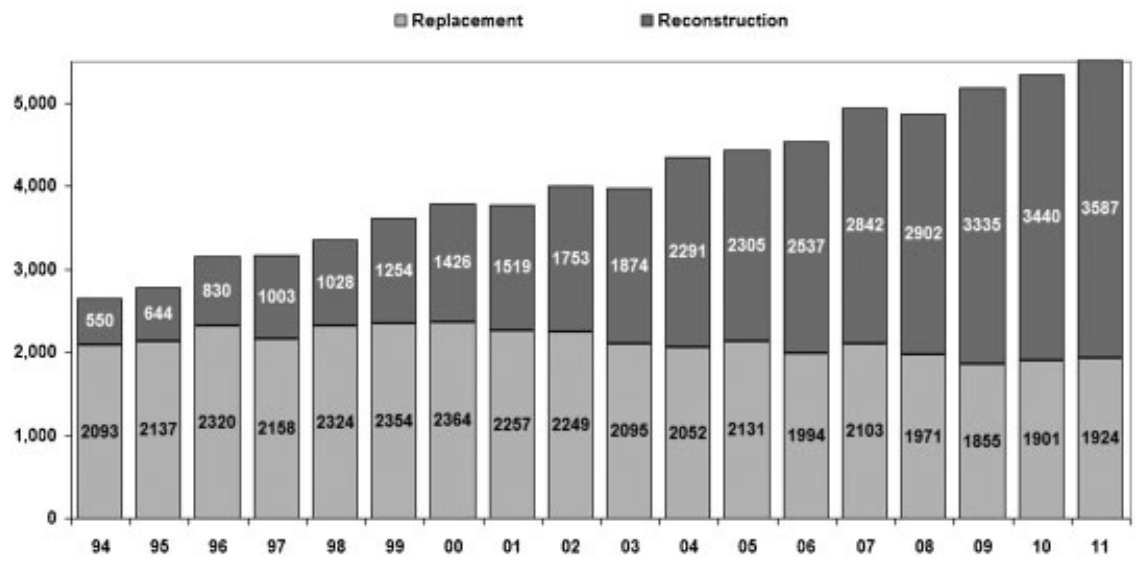

Figure 9 Isolated mitral valve surgery over the past 18 years. More reconstructions (64\%) than replacements (36\%) were performed. In 1994, the percentage of reconstructions was only $21 \%$. Due to the data collection method which is based on procedure codes, all isolated mitral valve procedures regardless of diagnosis, morphology, or urgency type are included. The rate of valve reconstruction would certainly be higher if patients with mitral valve stenosis, severe calcification, or endocarditis would have been excluded as it has been done in other publications, for example, Gammie et al.

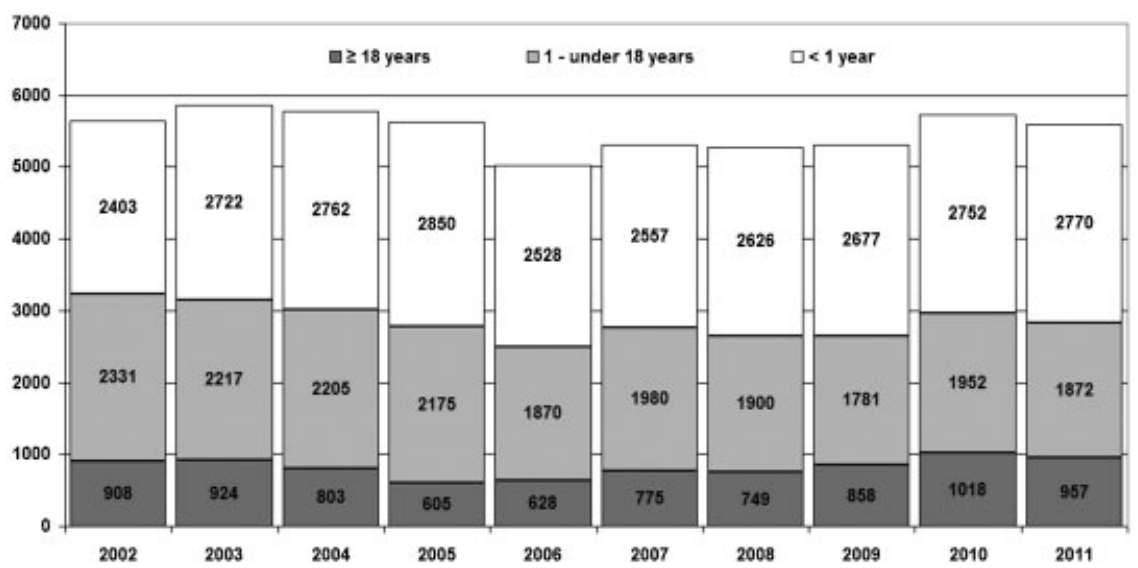

Figure 10 Development of congenital heart surgery in Germany over the past 10 years. Numbers remain more or less stable across previous years. However, there may be a bias since not all procedures for congenital heart surgery are necessarily counted in patients older than 18 years (e.g., aortic valve surgery).

LVAD Implantation

BVAD Implantation
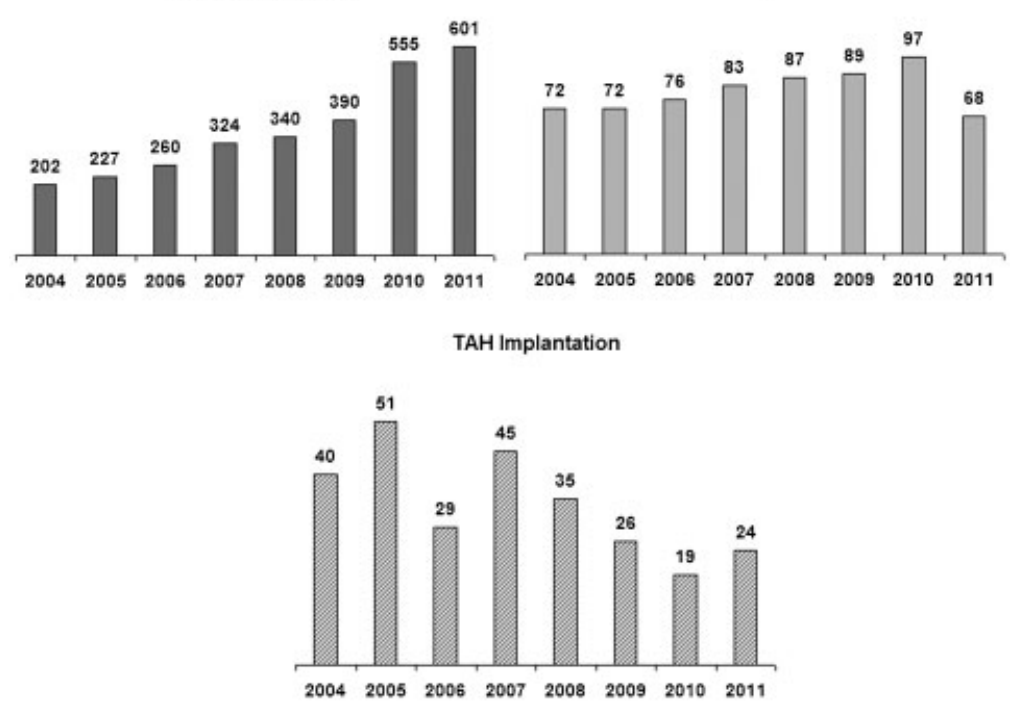

Figure 11 Development of mechanical circulatory support in Germany over the past 8 years. There is a significant increase in implantations of left ventricular assist devices (LVAD). However, in 2011, the number of implanted paracorporal biventricular support systems (BVAD) was only 70\% compared with the previous year. The number of total artificial heart implantations (TAH) is still low. 


\section{Acknowledgments}

On behalf of the German Society for Thoracic and Cardiovascular Surgery, the authors would like to thank the chairmen and their coworkers of all heart surgery units in Germany for their continuous cooperation and support for realizing this registry report.

\section{References}

1 Kalmar P, Irrgang E. Cardiac surgery in the Federal Republic of Germany during 1989. A report by the German Society for Thoracic and Cardiovascular Surgery. Thorac Cardiovasc Surg 1990;38(3):198-200

2 Kalmar P, Irrgang E. Cardiac surgery in the Federal Republic of Germany during 1990. A report by the German Society for Thoracic and Cardiovascular Surgery. Thorac Cardiovasc Surg 1991;39(3):167-169

3 Kalmar P, Irrgang E. Cardiac surgery in Germany during 1991. A report by the German Society for Thoracic and Cardiovascular Surgery. Thorac Cardiovasc Surg 1992;40(3):163-165

4 Kalmar P, Irrgang E. Cardiac surgery in Germany during 1992. A report by the German Society for Thoracic and Cardiovascular Surgery. Thorac Cardiovasc Surg 1993;41(3):202-204

5 Kalmar P, Irrgang E. Cardiac surgery in Germany during 1993. A report by the German Society for Thoracic and Cardiovascular Surgery. Thorac Cardiovasc Surg 1994;42(3):194-196

6 Kalmar P, Irrgang E. Cardiac surgery in Germany during 1994. A report by the German Society for Thoracic and Cardiovascular Surgery. Thorac Cardiovasc Surg 1995;43(3):181-183

7 Kalmár P, Irrgang E. Cardiac surgery in Germany during 1995. A report by the German Society for Thoracic and Cardiovascular Surgery. Thorac Cardiovasc Surg 1996;44(3):161-164

8 Kalmár P, Irrgang E. Cardiac surgery in Germany during 1996. A report by the German Society for Thoracic and Cardiovascular Surgery. Thorac Cardiovasc Surg 1997;45(3):134-137

9 Kalmár P, Irrgang E. Cardiac surgery in Germany during 1997. A report by the German Society for Thoracic and Cardiovascular Surgery. Thorac Cardiovasc Surg 1998;46(5):307-310

10 Kalmàr P, Irrgang E. Cardiac surgery in Germany during 1998. A report by the German Society for Thoracic and Cardiovascular Surgery. Thorac Cardiovasc Surg 1999;47(4):260-263

11 Kalmár P, Irrgang E. Cardiac surgery in Germany during 1999. Thorac Cardiovasc Surg 2000;48(4):XXVII-XXX

12 Kalmár P, Irrgang E. Cardiac surgery in Germany during 2001: a report by the German Society for Thoracic and Cardiovascular Surgery. Thorac Cardiovasc Surg 2002;50(6):30-35
13 Kalmár P, Irrgang E; German Society for Thoracic and Cardiovascular Surgery. Cardiac surgery in Germany during 2002: a report by German Society for Thoracic and Cardiovascular Surgery. Thorac Cardiovasc Surg 2003;51(5):25-29

14 Kalmár P, Irrgang E; German Society for Thoracic and Cardiovascular Surgery. Cardiac surgery in Germany during 2003: a report by the German Society for Thoracic and Cardiovascular Surgery. Thorac Cardiovasc Surg 2004;52(5):312-317

15 Gummert JF, Funkat A, Krian A. Cardiac surgery in Germany during 2004: a report on behalf of the German Society for Thoracic and Cardiovascular Surgery. Thorac Cardiovasc Surg 2005;53(6): 391-399

16 Gummert JF, Funkat A, Beckmann A, Hekmat K, Ernst M, Krian A. Cardiac surgery in Germany during 2005: a report on behalf of the German Society for Thoracic and Cardiovascular Surgery. Thorac Cardiovasc Surg 2006;54(5):362-371

17 Gummert JF, Funkat A, Beckmann A, et al. Cardiac surgery in Germany during 2006: a report on behalf of the German Society for Thoracic and Cardiovascular Surgery. Thorac Cardiovasc Surg 2007;55(6):343-350

18 Gummert JF, Funkat A, Beckmann A, et al; German Society for Thoracic and Cardiovascular Surgery. Cardiac surgery in Germany during 2007: a report on behalf of the German Society for Thoracic and Cardiovascular Surgery. Thorac Cardiovasc Surg 2008;56(6): 328-336

19 Gummert JF, Funkat A, Beckmann A, et al. Cardiac surgery in Germany during 2008. A report on behalf of the German Society for Thoracic and Cardiovascular Surgery. Thorac Cardiovasc Surg 2009;57(6):315-323

20 Gummert JF, Funkat A, Beckmann A, et al. Cardiac surgery in Germany during 2009. A report on behalf of the German Society for Thoracic and Cardiovascular Surgery. Thorac Cardiovasc Surg 2010;58(7):379-386

21 Gummert JF, Funkat AK, Beckmann A, et al. Cardiac surgery in Germany during 2010: a report on behalf of the German Society for Thoracic and Cardiovascular Surgery. Thorac Cardiovasc Surg 2011;59(5):259-267

22 Gammie JS, Zhao Y, Peterson ED, O’Brien SM, Rankin JS, Griffith BP. J. Maxwell Chamberlain Memorial Paper for adult cardiac surgery. Less-invasive mitral valve operations: trends and outcomes from the Society of Thoracic Surgeons Adult Cardiac Surgery Database. Ann Thorac Surg 2010;90(5):1401-1408, 1410, e1, discussion 1408-1410

23 Herbert MA, Prince SL, Williams JL, Magee MJ, Mack MJ. Are unaudited records from an outcomes registry database accurate? Ann Thorac Surg 2004;77(6):1960-1964, discussion 1964-1965 